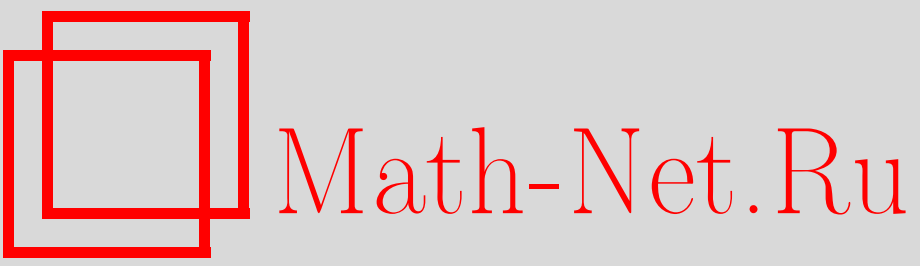

З. И. Шарифзода, Э. М. Мухамадиев, И. Д. Нуров, О циклических решениях уравнения Понтрягина с малым параметром, Итоги науки и техн. Сер. Соврем. мат. и ее прил. Темат. обз., 2021, том 194, 167-171

DOI: https://doi.org/10.36535/0233-6723-2021-194-167-171

Использование Общероссийского математического портала Math-Net.Ru подразумевает, что вы прочитали и согласны с пользовательским соглашением

http://www.mathnet.ru/rus/agreement

Параметры загрузки:

IP : 3.85 .7 .115

26 апреля 2023 г., 13:11:53 


\title{
О ЦИКЛИЧЕСКИХ РЕШЕНИЯХ УРАВНЕНИЯ ПОНТРЯГИНА С МАЛЫМ ПАРАМЕТРОМ
}

\author{
(c) 2021 г. З. И. ШАРИФЗОДА, Э. М. МУХАМАДИЕВ, И. Дж. НУРОВ
}

\begin{abstract}
АннотАция. Работа посвящена вопросам существования периодических решений систем нелинейных уравнений с малым параметром. Найдены условия существования периодических решений, которые существенно расширяют область применимости метода малого параметра Л. С. Понтрягина.
\end{abstract}

Ключевые слова: дифференциальное уравнение, периодическое решение, топологические методы, вращение векторного поля.

\section{CYCLIC SOLUTIONS OF THE PONTRYAGIN EQUATION WITH A SMALL PARAMETER}

\author{
(c) 2021 Z. I. SHARIFZODA, E. M. MUHAMADIEV, I. Dzh. NUROV
}

\begin{abstract}
In this paper, we examine the existence of periodic solutions to systems of nonlinear equations with a small parameter. We find conditions for the existence of periodic solutions, which expand the range of applicability of Pontryagin's method of small parameter.
\end{abstract}

Keywords and phrases: differential equation, periodic solution, topological methods, rotation of a vector field.

AMS Subject Classification: $34 \mathrm{~A} 34$

1. Постановка задачи и формулировка основных результатов. Рассмотрим систему

$$
\left\{\begin{array}{l}
\dot{x}=-y+\mu \cdot P(x, y, \mu) \\
\dot{y}=x+\mu \cdot Q(x, y, \mu)
\end{array}\right.
$$

где функции $P(x, y, \mu), Q(x, y, \mu)$ непрерывны по совокупности переменных $(x, y, \mu)$ в области $|\mu|<\mu_{0},(x, y) \in \mathbb{R}^{2}$. В работе изучается вопрос о существовании периодического решения цикла системы $(1)$ при $\mu \neq 0$. По функциям $P(x, y, 0), Q(x, y, 0)$ определим скалярную функцию

$$
F(r)=\int_{0}^{2 \pi}(\cos \varphi \cdot P(r \cos \varphi, r \sin \varphi, 0)+\sin \varphi \cdot Q(r \cos \varphi, r \sin \varphi, 0)) d \varphi .
$$

Для исследования вопроса о существовании циклов системы (1) отправным пунктом для авторов послужила классическая теорема Л. С. Понтрягина (см. [7]), которая сформулирована при предположении аналитичности функций $P(x, y, \mu), Q(x, y, \mu)$ по совокупности переменных $(x, y, \mu)$ (см. [1, с. 214]).

Теорема 1. Если для некоторого $\rho=\rho_{0}$ выполняются условия $F\left(\rho_{0}\right)=0, F^{\prime}\left(\rho_{0}\right) \neq 0$, то существуют такие числа $\varepsilon>0 u \delta>0$, что: 
(i) для любого $\mu,|\mu|<\delta$, система (1) имеет в $\varepsilon$-окрестности кривой $x^{2}+y^{2}=\rho_{0}$ один и только один предельный иикл, причем при $\mu \rightarrow 0$ он стягивается $\kappa$ окружности $x^{2}+y^{2}=\rho_{0}$ (являющейся траекторией системы (1) при $\mu=0$ );

(ii) этот предельный цикл является грубым предельным ииклом, устойчивым, когда $\mu$. $F^{\prime}\left(\rho_{0}\right)<0$, и неустойчивым, когда $\mu \cdot F^{\prime}\left(\rho_{0}\right)>0$.

Функция (2) определена и непрерывна, если функции $P(x, y, \mu), Q(x, y, \mu)$ лишь непрерывны по совокупности переменных. Но это условие не гарантирует дифференцируемости функции $F(r)$. В связи с этим возникает вопрос: как сформулировать утверждение, содержащее аналог теоремы Л. С. Понтрягина или аналог какой-либо ее части для случая непрерывных функций $P(x, y, \mu)$, $Q(x, y, \mu)$ ?

В настоящей работе сделана попытка ответить на сформулированный вопрос, и получены теоремы существования цикла у системы в условиях непрерывности функций $P(x, y, \mu), Q(x, y, \mu)$ в терминах самой функции $F(r)$. А именно, справедливы следующие теоремы.

Теорема 2. Пусть $\rho_{0}>0$-решение уравнения $F(\rho)=0$ и в окрестности точки $\rho_{0}\left[\rho_{0}-\right.$ $\left.\varepsilon_{0}, \rho_{0}+\varepsilon_{0}\right], \rho_{0}-\varepsilon_{0}>0$, функиия $F(\rho) \neq 0$ при $\rho \neq \rho_{0}$, причем значения функиии $F(\rho)$ меняют знак при переходе через точку $\rho_{0}$. Тогда система (1) при достаточно малых значениях $\mu$ имеет периодическое решение $(x(t, \mu), y(t, \mu))$, удовлетворяющее условию

$$
\left|\sqrt{x^{2}(t, \mu)+y^{2}(t, \mu)}-\rho_{0}\right|<\varepsilon_{0} .
$$

Теорема 3. Пусть существуют такие числа $0<\rho_{1}<\rho_{2}$, что $F\left(\rho_{2}\right) \cdot F\left(\rho_{1}\right)<0$. Тогда система (1) при достаточно малых значениях $\mu$ имеет периодическое решение $(x(t, \mu), y(t, \mu))$, удовлетворяющее условию

$$
\rho_{1}<\sqrt{x^{2}(t, \mu)+y^{2}(t, \mu)}<\rho_{2} .
$$

Доказательства теорем 2 и 3 основаны на применении топологических методов: вычисления вращения соответствующих вполне непрерывных векторных полей, нули которых определяют периодическое решение системы (1) (см., например, [2-6]).

2. Необходимое условие существования цикла. Пусть $(x(t), y(t))$ - решение системы $(1)$, не обращающееся в нуль. Полагая

$$
\left\{\begin{array}{l}
x(t)=r(t) \cos \varphi(t), \\
y(t)=r(t) \sin \varphi(t),
\end{array} \quad 0 \leqslant \varphi(0) \leqslant 2 \pi,\right.
$$

т.е. переходя к полярным координатам, получаем

$$
\left\{\begin{array}{l}
\dot{r}=\mu \cdot(P \cdot \cos \varphi+Q \cdot \sin \varphi), \\
r \cdot \dot{\varphi}=r+\mu \cdot(Q \cdot \cos \varphi-P \cdot \sin \varphi) .
\end{array}\right.
$$

Если для решения $(x(t), y(t))$ системы $(1)$ при данном значении $\mu$ правая часть второго уравнения системы (3) положительна при всех $t$, то время $t$ можно выразить через полярный угол $\varphi$ : $t=T(\varphi)$. Относительно функции $\rho(\varphi)=r(T(\varphi))$ от системы (3) перейдем к скалярному уравнению

где

$$
\frac{d \rho}{d \varphi}=\mu \cdot G(\rho, \varphi, \mu)
$$

$$
G(\rho, \varphi, \mu)=\frac{\rho \cdot(P \cdot \cos \varphi+Q \cdot \sin \varphi)}{\rho+\mu \cdot(Q \cdot \cos \varphi-P \cdot \sin \varphi)} .
$$

При этих предположениях справедлива следующее утверждение.

Лемма. Решение $(x(t), y(t))$ системы (1) при данном значении н является периодическим, m.е. $(x(t+\omega), y(t+\omega))=(x(t), y(t)), \omega=\omega(\mu)>0$, тогда и только тогда, когда соответствующее решение $\rho(\varphi)=r(T(\varphi))$ скалярного уравнения (4) является $2 \pi$-периодическим, т.е. $\rho(\varphi+2 \pi)=$ $\rho(\varphi)$. 
Теорема (необходимое условие). Предположим, что для некоторой последовательности значений $\mu=\mu_{k} \neq 0, \mu_{k} \rightarrow 0$ при $k \rightarrow \infty$, система (1) имеет периодическое решение $\left(x_{k}(t), y_{k}(t)\right)$ с периодом $\omega_{k}>0$, удовлетворяюоие условию

$$
M_{0} \leqslant \sqrt{x_{k}^{2}(t)+y_{k}^{2}(t)} \leqslant M, \quad M_{0}>0 .
$$

Тогда существует такое $\rho_{0} \in\left[M_{0}, M\right]$, что $F\left(\rho_{0}\right)=0$.

Доказательство. Пусть $\rho_{k}(\varphi)-2 \pi$-периодическое решение скалярного уравнения (4), соответствующее решению $\left(x_{k}(t), y_{k}(t)\right)$ системы (1) при $\mu=\mu_{k}$. Подставляя это решение в (4) и полагая $\mu=\mu_{k}$, затем интегрируя полученное тождество на промежутке $[0,2 \pi]$, в силу условии $\mu_{k} \neq 0$ получим

$$
\int_{0}^{2 \pi} G\left(\rho_{k}(\varphi), \varphi, \mu_{k}\right) d \varphi=0
$$

В силу условия теоремы последовательность функций $\rho_{k}(\varphi)$ равномерно ограниченна, а в силу уравнения (4) она равностепенно непрерывна и, более того, $\rho_{k}(\varphi)-\rho_{k}(0) \rightarrow 0$ при $k \rightarrow \infty$ равномерно на промежутке $[0,2 \pi]$. В силу теоремы Вейерштрасса без ограничения общности можно считать, что числовая последовательность $\rho_{k}(0)$ при $k \rightarrow \infty$ сходится к некоторому числу $\rho_{0} \in\left[M_{0}, M\right]$. В силу непрерывности функций $P(x, y, \mu), Q(x, y, \mu)$, и условия $\mu_{k} \rightarrow 0$, последовательность функций $G\left(\rho_{k}(\varphi), \varphi, \mu_{k}\right)$ при $k \rightarrow \infty$ равномерно сходится к функции

$$
G\left(\rho_{0}, \varphi, 0\right)=P\left(\rho_{0} \cos \varphi, \rho_{0} \sin \varphi, 0\right) \cdot \cos \varphi+Q\left(\rho_{0} \cos \varphi, \rho_{0} \sin \varphi, 0\right) \cdot \sin \varphi .
$$

Теперь, переходя к пределу в равенстве (5), получаем

$$
F\left(\rho_{0}\right)=\int_{0}^{2 \pi}\left(\cos \varphi \cdot P\left(\rho_{0} \cos \varphi, \rho_{0} \sin \varphi, 0\right)+\sin \varphi \cdot Q\left(\rho_{0} \cos \varphi, \rho_{0} \sin \varphi, 0\right)\right) d \varphi=0 .
$$

Теорема доказана.

3. Доказательство теоремы 2. В пространстве непрерывных функций $C[0,2 \pi]$ рассмотрим область

$$
\Omega=\left\{\rho(\varphi):\left|\rho(\varphi)-\rho_{0}\right|<\varepsilon_{0}, 0 \leqslant \varphi \leqslant 2 \pi\right\},
$$

где $\varepsilon_{0}<\rho_{0}$. На границе $\dot{\Omega}$ области $\Omega$ введем в рассмотрение семейство вполне непрерывных векторных полей

$$
\begin{aligned}
& \left(\Phi_{\mu} \rho\right)(\varphi)=\rho(\varphi)-\rho(2 \pi)-\mu \int_{0}^{\varphi} G(\rho(\psi), \psi, \mu) d \psi, \\
& \left(\Psi_{\mu} \rho\right)(\varphi)=\rho(\varphi)-\rho(2 \pi)-\mu \int_{0}^{\varphi} F(\rho(\psi)) d \psi .
\end{aligned}
$$

Отметим, что нули поля $\Phi_{\mu}$ являются $2 \pi$-периодическими решениями уравнения (4).

Покажем, что векторные поля (6), (7) при малых значениях $\mu \neq 0$ на границе $\dot{\Omega}$ области $\Omega$ не имеют нулевых векторов и являются гомотопными. Для этого достаточно показать, что семейство векторных полей

$$
\left(\Phi_{\mu, \lambda} \rho\right)(\varphi)=\rho(\varphi)-\rho(2 \pi)-\mu \lambda \int_{0}^{\varphi} G(\rho(\psi), \psi, \mu) d \psi-\mu(1-\lambda) \int_{0}^{\varphi} F(\rho(\psi)) d \psi
$$

при малых значениях $\mu \neq 0$ и при всех $\rho \in \dot{\Omega}$ и всех $\lambda \in[0,1]$ не обращается в нуль. Действительно, если предположить противное, то существуют такие последовательности $\mu=\mu_{k} \neq 0, \mu_{k} \rightarrow 0$, 
$\lambda_{k} \in[0,1]$ и $\rho_{k} \in \dot{\Omega}$, что $\left(\Phi_{\mu, \lambda} \rho\right)(\varphi)=0$ при $\mu=\mu_{k}, \lambda=\lambda_{k}$ и $\rho=\rho_{k}$. Следовательно, функция $\rho=\rho_{k}(\varphi)$ является $2 \pi$-периодическим решением дифференциального уравнения

$$
\frac{d \rho}{d \varphi}=\mu \lambda \cdot G(\rho, \varphi, \mu)+\mu(1-\lambda) F(\rho)
$$

и удовлетворяет условию

$$
\lambda \int_{0}^{2 \pi} G(\rho(\psi), \psi, \mu) d \psi+(1-\lambda) \int_{0}^{2 \pi} F(\rho(\psi)) d \psi=0
$$

при $\mu=\mu_{k}, \lambda=\lambda_{k}$. В силу (8) для последовательности функций $\rho_{k}(\varphi)$ имеем $\rho_{k}(\varphi)-\rho_{k}(0) \rightarrow 0$ при $k \rightarrow \infty$ равномерно на промежутке $[0,2 \pi]$. Без ограничения общности можно считать, что последовательности $\rho_{k}(0) \rightarrow \rho_{*}, \lambda_{k} \rightarrow \lambda_{*}$ при $k \rightarrow \infty$. В равенстве $(9)$, полагая $\mu=\mu_{k}, \lambda=\lambda_{k}$ и $\rho(\varphi)=\rho_{k}(\varphi)$, затем перейдя к приделу при $k \rightarrow \infty$, получим

$$
\lambda_{*} \int_{0}^{2 \pi} G\left(\rho_{*}, \psi, 0\right) d \psi+\left(1-\lambda_{*}\right) \int_{0}^{2 \pi} F\left(\rho_{*}\right) d \psi=2 \pi F\left(\rho_{*}\right)=0 .
$$

С другой стороны, из условия $\rho_{k} \in \dot{\Omega}$ следует $\left|\rho_{*}-\rho_{0}\right|=\varepsilon_{0}$, что противоречит выбору $\varepsilon_{0}$. Полученное противоречие доказывает гомотопность векторных полей (6) и (7) на границе $\dot{\Omega}$ области $\Omega$ и справедливость равенства $\gamma\left(\Phi_{\mu}, \dot{\Omega}\right)=\gamma\left(\Psi_{\mu}, \dot{\Omega}\right)$ их вращений при достаточно малых значениях $\mu$.

Теперь покажем, что вращение вполне непрерывного векторного поля $\Psi_{\mu}$ на границе $\dot{\Omega}$ области $\Omega$ отлично от нуля. Рассмотрим семейство векторных полей

$$
\left(\Psi_{\lambda \mu} \rho\right)(\varphi)=\rho(\varphi)-\rho(2 \pi)-\mu \int_{0}^{\varphi} F(\rho(\psi)) d \psi-\mu \lambda \int_{\varphi}^{2 \pi} F(\rho(\psi)) d \psi, \quad 0 \leqslant \lambda \leqslant 1 .
$$

В частности, при $\lambda=0, \Psi_{0 \mu}=\Psi_{\mu}$, а при $\lambda=1$ имеем

$$
\left(\Psi_{1 \mu} \rho\right)(\varphi)=\rho(\varphi)-\rho(2 \pi)-\mu \int_{0}^{2 \pi} F(\rho(\psi)) d \psi
$$

Векторные поля $\left(\Psi_{\lambda \mu} \rho\right)(\varphi), \forall \lambda \in[0,1]$ при малых значениях $\mu$ на границе $\dot{\Omega}$ области $\Omega$ не имеют нулевых векторов и, следовательно, поля $\left(\Psi_{0 \mu} \rho\right)(\varphi)$ и $\left(\Psi_{1 \mu} \rho\right)(\varphi)$ являются гомотопными на $\dot{\Omega}$ и поэтому $\gamma\left(\Psi_{\mu}, \dot{G}\right)=\gamma\left(\Psi_{1 \mu}, \dot{G}\right)$.

Оператор

$$
\left(A_{\mu} \rho\right)(\varphi)=(2 \pi)+\mu \int_{0}^{2 \pi} F(\rho(\psi)) d \psi
$$

определяющий векторное поле $\Psi_{1 \mu}$, действует из пространства $C[0,2 \pi]$ в подпространство постоянных функций. Это подпространство обозначим через $E_{0}$ и положим $G=\Omega \cap E_{0}$. Очевидно, $G=\left(\rho_{0}-\varepsilon_{0}, \rho_{0}+\varepsilon_{0}\right)$. Согласно лемме Лере-Шаудера (см. [4]) вращение вполне непрерывного векторного поля $\Psi_{1 \mu}$ на границе $\dot{\Omega}$ области $\Omega$ совпадет с вращением его сужения $\bar{\Psi}_{1 \mu}$ на границе $\dot{G}$ области $G$. Нетрудно видеть, что

$$
\bar{\Psi}_{1 \mu} \rho \equiv-\mu \cdot 2 \pi \cdot F(\rho), \quad \rho \in\left(\rho_{0}-\varepsilon_{0}, \rho_{0}+\varepsilon_{0}\right),
$$

и поэтому его вращение $\gamma\left(\bar{\Psi}_{1 \mu}, \dot{G}\right)$ на границе $\dot{G}$ области $G$ равно

$$
\gamma\left(\bar{\Psi}_{1 \mu}, \dot{G}\right)=-\operatorname{sign} \mu\left(\operatorname{sign} F\left(\rho_{0}+\varepsilon_{0}\right)-\operatorname{sign} F\left(\rho_{0}-\varepsilon_{0}\right)\right) / 2 .
$$

Следовательно, вращение $\gamma\left(\Phi_{\mu}, \dot{\Omega}\right)$ поля $\Phi_{\mu}$ на границе $\dot{\Omega}$ области $\Omega$ отлично от нуля. Отсюда следует существование $2 \pi$-периодического решения уравнения (4) в области $\Omega$. Теорема 2 доказана.

Доказательство теоремы 3 проводится аналогично доказательству теоремы 2. 


\section{СПИСОК ЛИТЕРАТУРЫ}

1. Баутин Н. Н., Леонтович Е. А. Методы и приемы качественного исследования динамических систем на плоскости. - М.: Наука, 1976.

2. Красносельский M. A. Топологические методы в теории нелинейных интегральных уравнений. - М.: ГТТИ, 1956.

3. Красносельский M. А. Оператор сдвига по траекториям дифференциальных уравнений. - М.: Наука, 1966.

4. Красносельский М. А., Забрейко П. П. Геометрические методы нелинейного анализа. - М.: Наука, 1975.

5. Красносельский М. А., Перов А. И., Поволоцкий А. И., Забрейко П. П. Векторные поля на плоскости. - М.: Физматгиз, 1963.

6. Мухамадиев Э. М. К теории периодических решений систем обыкновенных дифференциальных уранений// Докл. АН СССР. - 1970. - 194, № 3. - С. 510-513.

7. Pontryagin L. S. Über Autoschwingungssysteme, die den Hamiltonschen nahe liegen// Phys. Z. Sowjetunion. - 1934. - 6. - P. 25-28.

Шарифзода 3. И.

Таджикский национальный университет, Душанбе, Таджикистан

E-mail: sakhara-2803@mail.ru

Мухамадиев Эргашбой Мирзоевич

Вологодский государственный университет

E-mail: emuhamadiev@rambler.ru

Нуров Исхокбой Джумаевич

Таджикский национальный университет, Душанбе, Таджикистан

E-mail: nid1@mail.ru 